\title{
Mitigating myopia in tuberculosis
}

\author{
C-12, a cluster of CD4+ $T_{H}$ 17-like cells, defined by unbiased multimodal profiling of memory $T$ cells, is significantly \\ reduced in patients who have recovered from TB (progressors) as compared to those who were infected but did not \\ develop the disease (non-progressors).
}

\section{Sarah J. Dunstan and Thomas R. Hawn}

n nvisibility haunts humanity and prevents scientific progress. Prior to the discovery of the etiology of consumption, debates raged about its origins and treatments. New tools settled the debate in 1882, when Robert Koch used new staining methods and visualized the Mycobacterium tuberculosis (Mtb) bacillus for the first time with a microscope $e^{1,2}$. This discovery ushered in a new era that eventually led to effective tuberculosis (TB) treatments in the 1950s. Over 130 years later, TB's global impact remains sobering, with approximately 10 million people falling ill and 1.4 million dying annually ${ }^{3}$. Two frequently asked questions regarding $\mathrm{TB}$ are: why do only some people who encounter $M t b$ develop TB disease, and why do we still lack a highly effective vaccine? Despite decades of multidisciplinary research to discover immunologic correlates and mechanisms of TB disease progression and vaccine protection, the answers remain elusive. The TB field continues to strive to reveal the invisible to generate immunologic insights that can be harnessed for new and effective vaccine strategies ${ }^{4,5}$. Equipped with new tools, Nathan and colleagues ${ }^{6}$ endeavor to address these questions in the current issue of Nature Immunology. Using state-of-the art immunologic profiling methods, the authors identified a unique $\mathrm{T}$ cell memory state that could in part contribute to an individual's predisposition to TB disease progression.

To overcome previous limitations in $\mathrm{T}$ cell profiling, the study by Nathan et al. had three crucial aspects to identify persistent interindividual immune cell differences underlying the risk of progression to active TB disease. First, the team utilized a comprehensively described TB cohort to enable the identification of demographic and environmental variables that confound $\mathrm{T}$ cell profiles ${ }^{7}$. Second, sampling to identify progression-associated memory $\mathrm{T}$ cells was not conducted during the inflamed disease state. Lastly, the use of CITE-seq (cellular indexing of transcriptomes and epitopes by sequencing) to capture both surface protein and transcriptional $\mathrm{T}$ cell profiles provided an unbiased integrated multimodal strategy that did not rely on any one class of $\mathrm{T}$ cell markers to capture and characterize the complexity of functional $\mathrm{T}$ cell states.

Interested in discovering differences in inherent immunity preceding progression to disease rather than differences resulting from acute disease-induced inflammation, Nathan et al. re-recruited healthy participants who had recovered from TB (progressors) and tuberculin skin testpositive household contacts who never progressed to active TB (non-progressors). They profiled 500,089 "steady-state" CD45memory $\mathrm{T}$ cells from these individuals by CITE-seq. Unsupervised clustering of surface protein and transcriptional markers defined $31 \mathrm{~T}$ cell states, with $\mathrm{CD} 4^{+}$ T cell clusters predominating. Age, sex, winter blood draw and European genetic ancestry were associated with memory $\mathrm{T}$ cell composition, so it was essential to adjust for the effect of these confounders in the analysis of $\mathrm{T}$ cell states and TB progression. C-12, a cluster of $\mathrm{CD}^{+}$type 17 helper $\mathrm{T}\left(\mathrm{T}_{\mathrm{H}} 17\right)$-like cells, was shown to be significantly reduced in TB progressors (Fig. 1). This $\mathrm{T}_{\mathrm{H}} 17$ cell subset cannot be defined by mRNA or surface protein markers alone; it has a $\mathrm{CD} 4^{+}$effector surface phenotype, lacks surface activation and exhaustion markers and has elevated $R O R C$ expression (the gene encoding the $\mathrm{T}_{\mathrm{H}} 17$-defining transcription factor) and reduced TBX21 (the $\mathrm{T}_{\mathrm{H}} 1$-defining transcription factor) and IFNG (encoding IFN- $\gamma$ ) expression. A surrogate of C-12 abundance was assessed in publicly available bulk expression data, using a $\mathrm{C}-12$ score as an estimate. Even though the C-12 score was not an exact proxy, it revealed that this $\mathrm{T}$ cell state was neither increased by latency nor decreased due to treatment, and reduced C-12 abundance may predate disease progression. To characterize the functionality of C-12, a minimal set of surface protein markers $\left(\mathrm{CD} 4{ }^{+} \mathrm{CD} 45 \mathrm{RO}^{+} \mathrm{CD} 26^{+} \mathrm{CD} 161^{+} \mathrm{CCR}^{+}\right)$ was used as a sortable $\mathrm{C}-12$ proxy. Upon stimulation, these relatively rare cells had a $\mathrm{T}_{\mathrm{H}}$ 17-like phenotype, producing a disproportionately large amount of interleukin (IL)-17A and IL-22 as compared to other $\mathrm{CD}^{+}$memory $\mathrm{T}$ cells and producing IFN $-\gamma$ at rates comparable to the whole $\mathrm{CD} 4{ }^{+}$compartment. However, in individuals who progressed to having active $\mathrm{TB}$, this $\mathrm{T}$ cell state was reduced not only in frequency but also in functionality, and it produced less IL-17 and IL-22.

Single-cell sequencing has provided a new lens to re-examine the helper $\mathrm{T}$ cell subset paradigm, identifying subtle variation within known subsets and determining new cell states. A recent paper $^{8}$ showed that in response to various pathogens, $\mathrm{CD}^{+}$effector T cells represented a continuum of polarized phenotypes, which could not easily be divided into discrete helper $\mathrm{T}$ cell lineages. The unsupervised clustering of cell states reported by Nathan et al. also illustrated this, showing that $\mathrm{T}_{\mathrm{H}} 1$ and $\mathrm{T}_{\mathrm{H}} 17$ cells are a heterogeneous continuum. The utility of CITE-seq ${ }^{9}$ in indexing transcriptomes and protein markers at the single-cell level provides practical accessibility to highly defined cell states by using a closely related proxy of protein surface markers. This allows both the downstream functional analysis of this specific and potentially protective cell state as well as the interrogation of public bulk expression databases of cohorts with various TB phenotypes. One such database from South Africa previously implicated $\mathrm{T}_{\mathrm{H}} 17$ cell suppression in TB disease progressors ${ }^{10}$. Nathan et al. used the C-12 score trained on T cells and PBMCs to show that the relatively rare $\mathrm{C}-12 \mathrm{~T}_{\mathrm{H}} 17$ subset was $9 \%$ lower in active cases prior to progression as compared to $M t b$-infected controls in South Africa, providing further evidence of the involvement of this cell state in TB disease progression.

For many years, inducing IFN- $\gamma$ has been among the highest priority immunologic goals for vaccine development ${ }^{4}$. Although IFN- $\gamma$ is important for protective immune responses to $M t b$, the focus on it has fostered myopia. Approaches that are less 


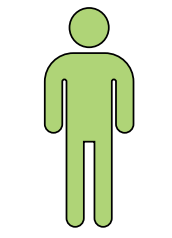

TB progressors

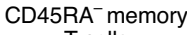
$\mathrm{T}$ cells

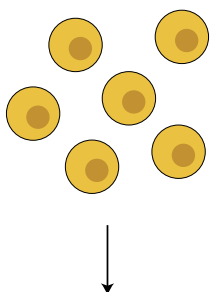

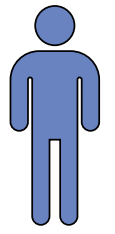

TB non-progressors

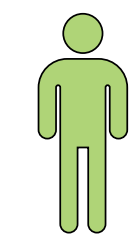

TB progressors

CITE-seq identified 31 multimodal clusters (C-1 to $\mathrm{C}-31)$

$\mathrm{C}-12=\mathrm{CD}^{+} \mathrm{CD} 45 \mathrm{RO}^{+} \mathrm{CD}^{2} 6^{+} \mathrm{CD} 161^{+} \mathrm{CCR}^{+} \mathrm{T}_{\mathrm{H}}$ 17-like subset
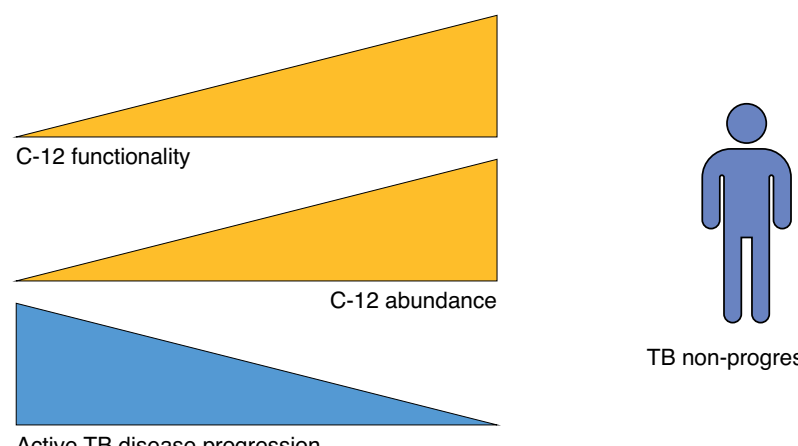

TB non-progressors

Active TB disease progression

Fig. 1 | C-12 cell numbers are significantly reduced in TB progressors as compared to TB non-progressors. Unbiased multimodal profiling (CITE-seq) of memory $T$ cells from patients who have recovered from TB (TB progressors) and from those who were infected but never developed the disease (TB

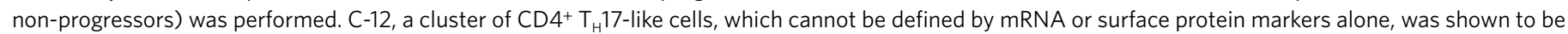
reduced in abundance and functionality in TB progressors.

biased and more comprehensive, such as those employed by Nathan et al., provide important new directions beyond IFN- $\gamma$. The identification of four demographic and environmental variables that confound $\mathrm{T}$ cell profiles in this study leads one to think about other unmeasured parameters that could have confounded the findings. What effect would the bacterial sequence variability of the infecting pathogen have on the $\mathrm{T}$ cell profiles? Would the microbiome or previous encounters with other pathogens (such as non-tuberculous mycobacteria) or vaccines (such as BCG) confound the profiling? What effect does trained immunity have on $\mathrm{C} 12$ states? If an inherent predisposition to $\mathrm{TB}$ disease progression is due to the reduced abundance and functionality of this T cell state, what modulates this genetically or epigenetically in an individual? Does it represent a stable cell state? Transcriptomic profiling of cell states is a snapshot in time, and in the study by Nathan et al., the analysis was conducted post-infection. More could be revealed by profiling the entire spectrum of TB clinical states, including the absence of classical evidence of infection following exposure (so-called resisters), during $M t b$ infection conversion and reversion, and after treatment of $M t b$ infection. More could also be revealed by adding further layers of information (for example, genomic or epigenetic) to exploit the full potential of data integration using an unbiased artificial intelligence approach.

Nathan et al. suggest that when people have an inherent reduced abundance of $\mathrm{C}-12$, it predisposes them to active TB disease. C-12 cells may therefore contribute to halting TB disease progression by producing a disproportionately large amount of IL-17A, IL-22 and IFN- $\gamma$ on exposure (stimulation) to $M t b$, but they must be at optimal abundance and functionality to take effect. Although a causal link between the $\mathrm{C}-12$ cell state and disease progression is yet to be established, functional analysis of this cell state may uncover specific antimycobacterial potential. By combining state-of-the art immunologic, genomic and bioinformatic tools with a rigorously designed clinical cohort, Nathan et al. have increased the visibility of the immunologic mechanisms underlying susceptibility to consumption. These critical insights will help to treat our collective TB mechanistic myopia and illuminate paths forward.

Sarah J. Dunstan (D) $1 \times$ and Thomas R. Hawn ${ }^{2}$ ${ }^{1}$ Peter Doherty Institute for Infection and Immunity, Department of Infectious Diseases, University of Melbourne, Victoria, Australia. ${ }^{2}$ Department of Medicine, University of Washington, Seattle,

WA, USA.

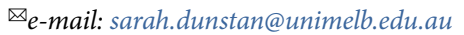

Published online: 24 May 2021

https://doi.org/10.1038/s41590-021-00935-z

References

1. Koch, R. Berliner Klin. Wochenschr. 15, 221-230 (1882).

2. Centers for Disease Control. MMWR Morb. Mortal. Wkly Rep. 31, 121-123 (1982); https://www.cdc.gov/mmwr/preview/ mmwrhtml/00000222.htm

3. Global Tuberculosis Report 2019 (WHO, 2020).

4. Sable, S. B., Posey, J. E. \& Scriba, T. J. Clin. Microbiol. Rev. 33, e00100-19 (2019).

5. Kaufmann, S. H. E. Front. Immunol. 11, 316 (2020).

6. Nathan, A. Nat. Immunol. https://doi.org/10.1038/s41590-02100933-1 (2021).

7. Becerra, M. C. et al. BMJ 367, 15894 (2019).

8. Kiner, E. et al. Nat. Immunol. 22, 216-228 (2021).

9. Stoeckius, M. et al. Nat. Methods 14, 865-868 (2017).

10. Scriba, T. J. et al. PLoS Pathog. 13, e1006687 (2017).

The authors declare no competing interests. 